\title{
Brain aggregoma with clonal B-cell perivascular proliferation detected by next-generation sequencing. A case report and review of the literature
}

\author{
Alenka Matjašič ${ }^{1}$, Karmen Wechtersbach ${ }^{1}$, Rajko Kavalar ${ }^{2}$, Matjaž Voršič ${ }^{3}$, Jernej Mlakar ${ }^{1}$, Janez Ravnik ${ }^{3}$, Mara Popović ${ }^{1}$ \\ ${ }^{1}$ Institute of Pathology, Faculty of Medicine, University of Ljubljana, Ljubljana, Slovenia, ${ }^{2}$ Department of Pathology, University \\ Medical Centre Maribor, Maribor, Slovenia, ${ }^{3}$ Department of Neurosurgery, University Medical Centre Maribor, Maribor, Slovenia
}

\begin{abstract}
Light-chain deposition disease ( $L C D D)$, a rare type of monoclonal immunoglobulin deposition disease, can be presented as systemic or localized, very rarely affecting central nervous system (CNS). Only 10 cases of CNS-LCDD have been described so far. We present an eleventh case of cerebral tumour-like LCDD, called aggregoma, and compare it with previously reported cases. A 49-year-old patient was admitted to the hospital due to a first generalized epileptic seizure. Magnetic resonance imaging (MRI) showed focal lesion in the right occipital lobe. Abundant parenchymal aggregates of pale eosinophilic material were observed, Congo red negative, Thioflavin $T$ moderately positive, and $\lambda$-light chain positive, but $\kappa$ negative in immunofluorescence with mild perivascular lymphoplasmacytic infiltrates in the intervening brain tissue. Clonality testing by next-generation sequencing showed the monoclonal nature of $B$-lymphocytes. Electron microscopy showed a finely granular ultrastructure of the aggregates without deposition in the vessel walls. A whole-body workup did not show any extra-cerebral immune dyscrasias.
\end{abstract}

Key words: aggregoma, CNS-LCDD, immunoglobulin light chains, next-generation sequencing, paraproteinaemia.

\section{Introduction}

Monoclonal immunoglobulin deposition disorders (MIDDs) are well-known clinicopathological entities related to the deposition of monoclonal immunoglobulins in fibrillar amyloidotic and, much more rarely, in granular or amorphous non-amyloidotic form (Randall type) [2]. Systemic MIDDs in both forms, light chain amyloidosis (AL), and, in non-amyloidotic form designated light and/or heavy chain deposition disease (LCDD/HCDD), always affect the kidneys, but other organs can be affected. The only functionally preserved organ in systemic MIDDs is the brain, due to the blood-brain barrier (BBB), with an exception to this rule [6].

Localized MIDDs have been described in respiratory and gastrointestinal tracts, lymph nodes, bones, and brain, usually as tumour-like amyloid deposits designated amyloidomas or, much more rarely, as tumour-like non-amyloid deposits called aggregomas [19]. Diffuse LCDD restricted to a single organ has also been described [16]. Only 10 cases of LCDD restricted to the brain have so far been described

Communicating author:

Prof. Mara Popović, MD, PhD, Institute of Pathology, Faculty of Medicine, University of Ljubljana, 1000 Ljubljana, Slovenia,

e-mail: mara.popovic@mf.uni-lj.si 
$[1,4,5,10-13,15,17,18]$. The majority of them were aggregomas, with or without vascular involvement. Only 1 case manifested as multifocal cerebral light chain vasculopathy (CLCV) [13].

Herein we describe a female patient with brain aggregoma and further compare it with 10 reported cases.

\section{Case report}

The patient was admitted to the neurological department after a first generalized epileptic seizure without neurological deficits. Magnetic resonance imaging (MRI) showed a focal lesion in the right occipital lobe (Fig. 1). Surgical complete excision of a firm lesion, sharply demarcated from oedematous brain tissue, was performed. The postoperative head MRI revealed postoperative changes after complete excision of the lesion (Fig. 1).

The patient had no signs of systemic lymphoproliferative disease.

Microscopic examination revealed abundant multifocal homogenous material with crack-like artefacts (Fig. 2A), which was Congo red negative (Fig. 2B) but Thioflavin T moderately positive (Fig. 2C). Immunofluorescence on lambda $(\lambda)$ and kappa $(\kappa)$ light chain (LC) showed aggregates to be $\lambda$-positive (Fig. 2D) and $\kappa$-negative (figure not shown). Mild perivascular lymphoplasmacytic infiltrates in the intervening brain tissue were composed mostly of T- and B-lymphocytes and very few plasma cells with no $L C$ restriction (figures not shown). A small piece of formalin-fixed, paraf-
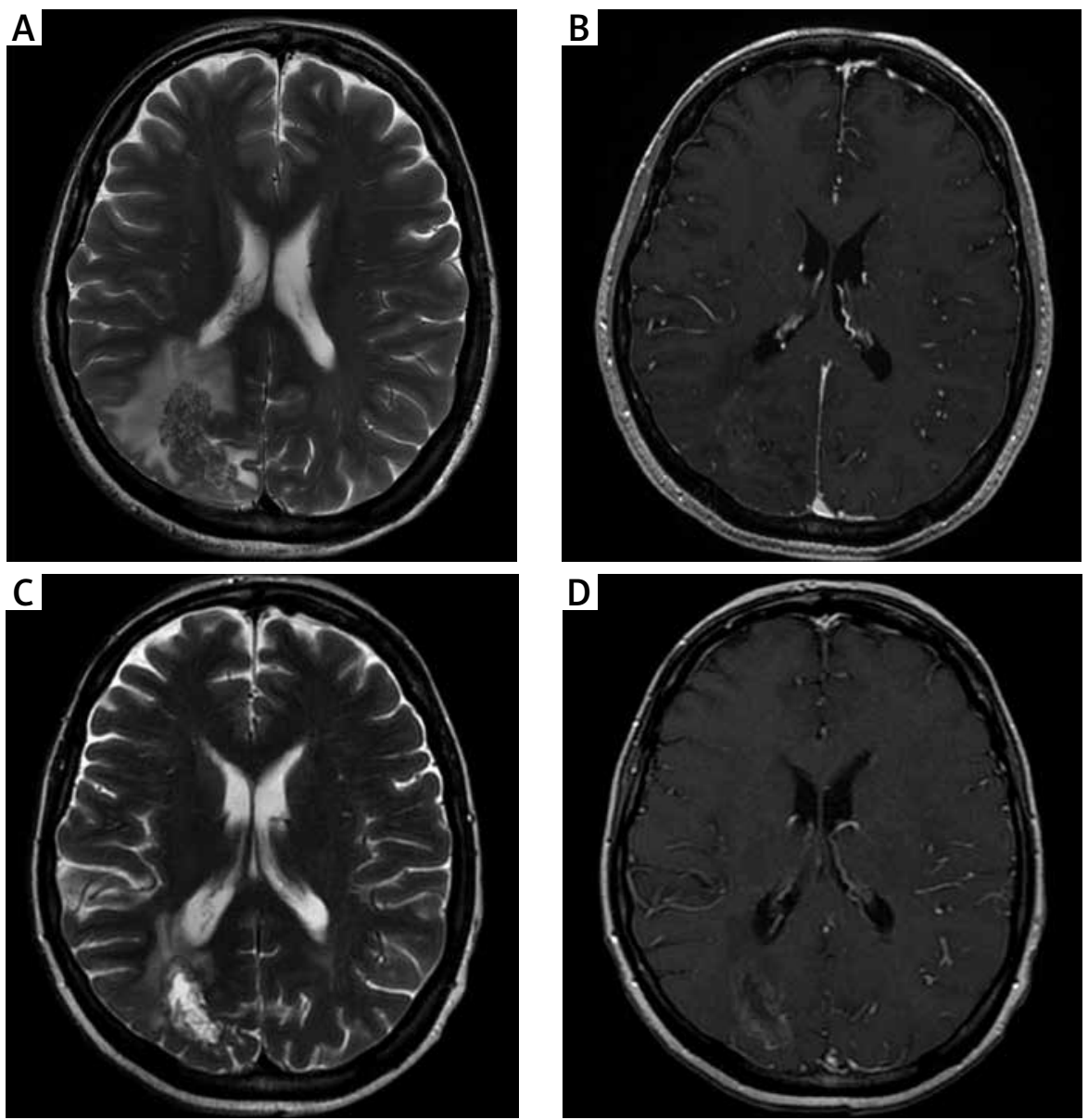

Fig. 1. Magnetic resonance imaging (MRI) of the brain before (A, B) and after surgery (C, D). A) The T2-weighted axial image shows a slightly unevenly hyper-intense lesion in the right occipital lobe, with surrounding oedema. B) The T1-weighted axial image with gadolinium shows very slight contrast enhancement of the lesion. C) The T2-weighted axial postoperative image shows macroscopically the completely removed lesion. D) No contrast enhancement on the T1-weighted image after the lesion has been removed. 

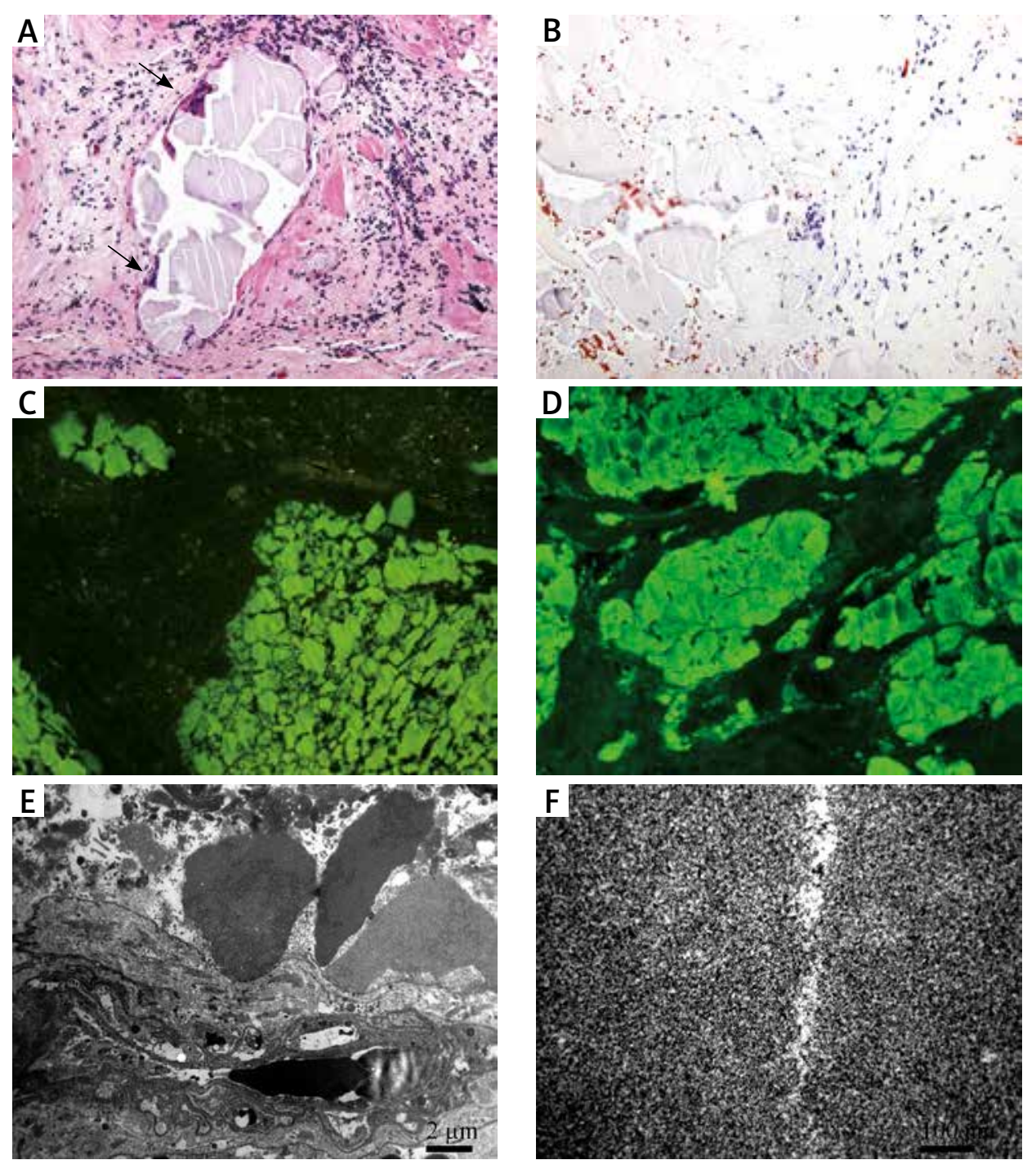

Fig. 2. Microscopic presentation of the lesion. A) Foci of homogenous eosinophilic deposits with some multinucleated giant cells (arrows) and some mononuclear cells in adjacent brain tissue. B) Deposits are Congo red negative but C) Thioflavin T moderately positive. D) Deposits are strongly positive for light chain immunofluorescence. E) Electron-dense rectangular deposits in brain tissue above a small artery but not in its wall. F) On higher magnification, the deposits are finely granular. Original magnification A-D 200x.

fin-embedded (FFPE) tissue was processed for electron microscopy, as previously described [7], revealing electron-dense finely granular material in the brain tissue but not in the vessel walls (Fig. 2E, F). A diagnosis of tumour-like LCDD or aggregoma was made.

We isolated total DNA from perivascular lymphocytic infiltrates, which were present between the abundant aggregates of $\lambda$-LC, enriched in the sample as previously described [9]. Next-generation sequencing (NGS)-based clonality testing of B-lymphocytes was performed using commercially available LymphoTrack assays (Invivoscribe Technologies) targeting immunoglobulin heavy $(\mathrm{IGH})$ and $\kappa$ light (IGK) chain, following the manufacturer's protocols. We detected a monoclonal B-lymphocyte population with rearrangements in IGH-FR2 and IGK (see Fig. 3), further supporting $\lambda$-LC deposits and the aggregoma diagnosis.

\section{Discussion with review of previously reported cases}

The presented case of cerebral LCDD is similar to some, but different from others, of 10 pre- 
A

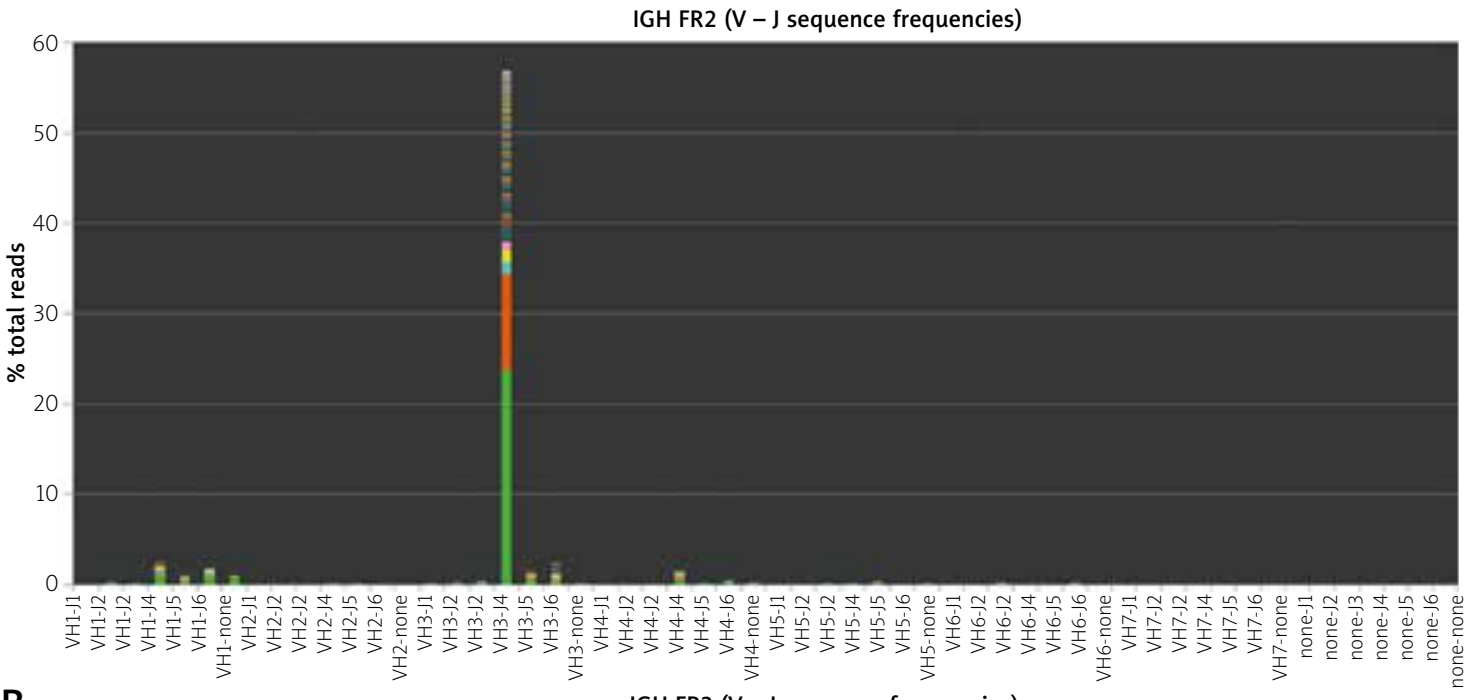

B

IGH FR3 (V - J sequence frequencies)

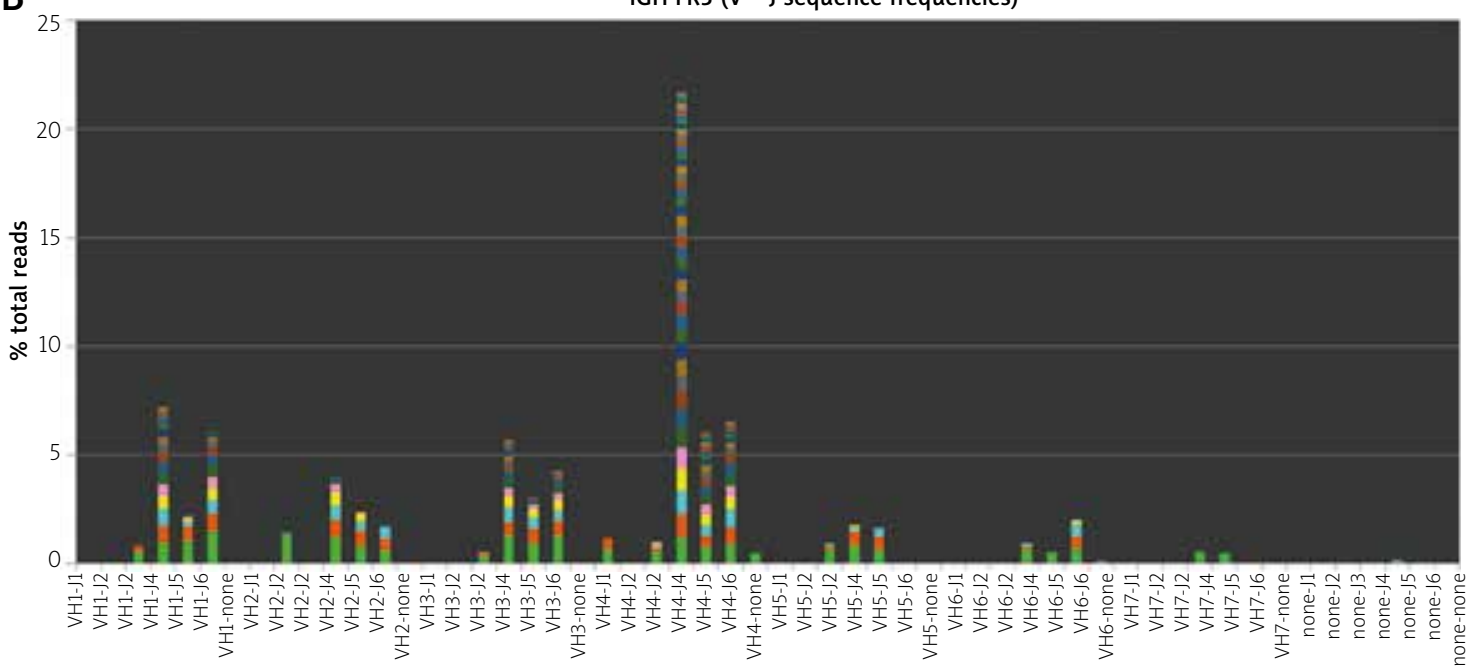

C

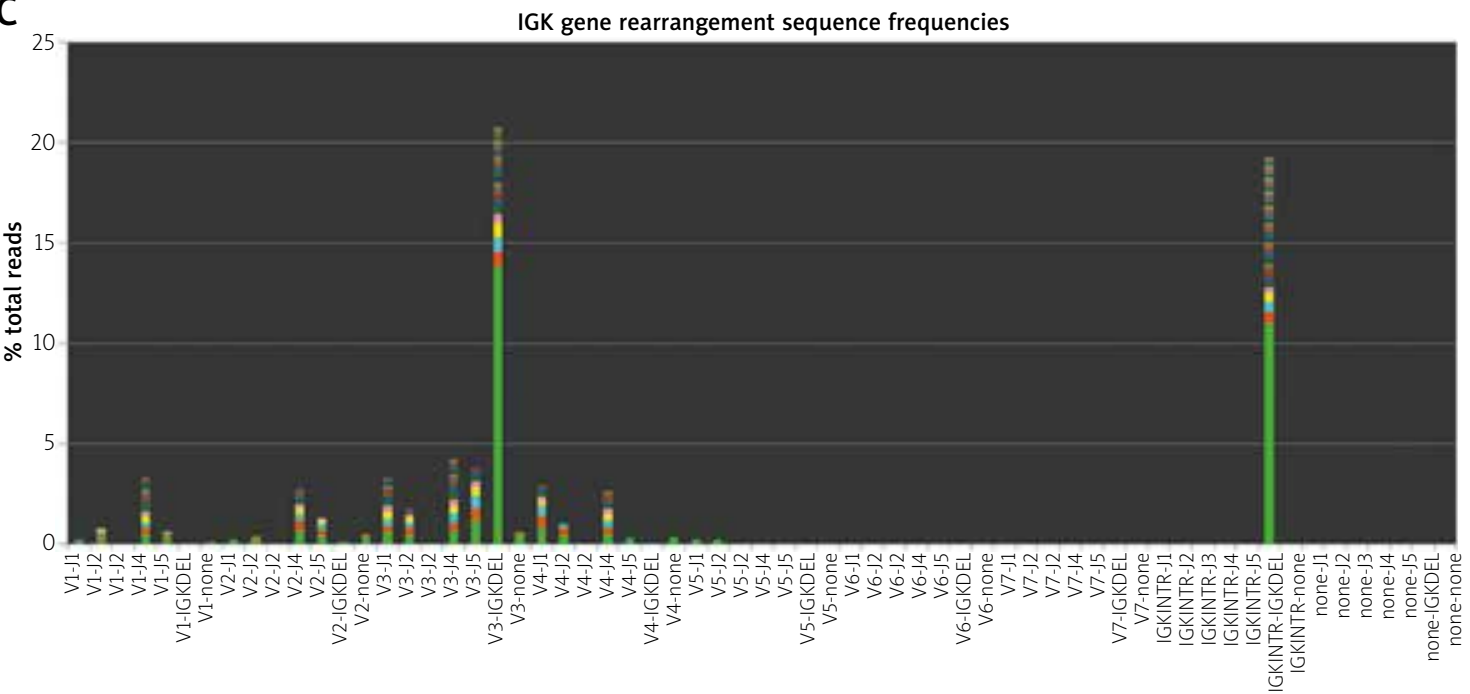

Fig. 3. Next-generation sequencing (NGS) for immunoglobulin heavy (IGH) chain and immunoglobulin $\kappa$ (IGK) light chain clonality detection. The NGS (using Invivoscribe assays and S5 Ion Torrent system) showed a monoclonal B-cell population. Each coloured box represents the frequency of reads with an identical sequence. NGS results show a monoclonal pattern in IGH regions FR2 (68343 reads) and IGK (403081 reads), and a polyclonal pattern in the IGH FR3 region (139975 reads). Sequencing of the FR1 region failed, because it did not produce the number of reads required for a reliable analysis (1363 reads, the threshold is set at 20000 reads). The 2 clones present in IGK analysis (V3/IGKDEL and IGKINTR/IGKDEL) (bottom histogram) suggest inactivation of IGK alleles and subsequent rearrangement of immunoglobulin $\lambda$ light chain genes. IGL expression requires a functional rearrangement of the IGL gene, which happens with inactivation of IGK alleles through deletion of their regulatory elements [14]. 


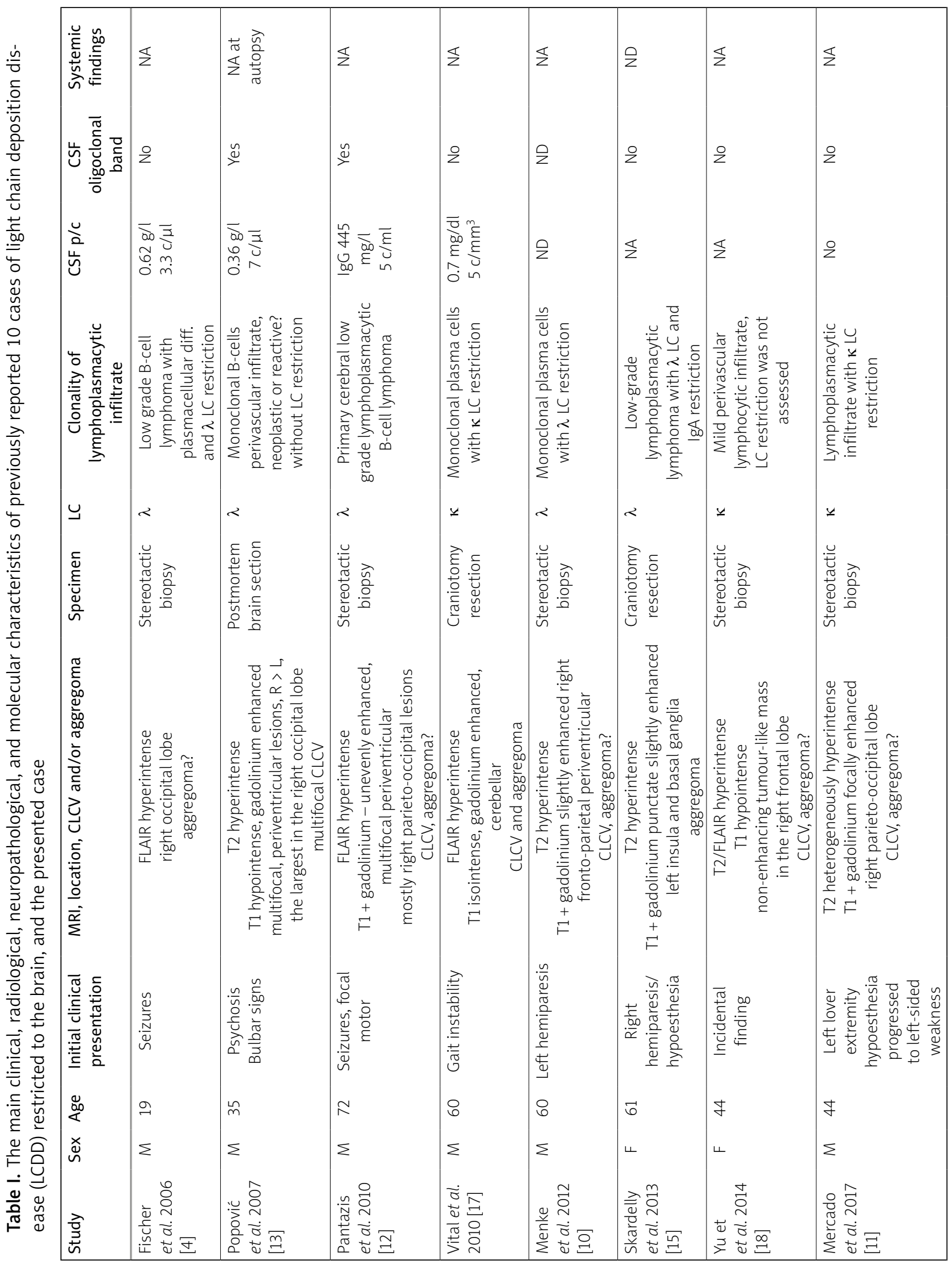




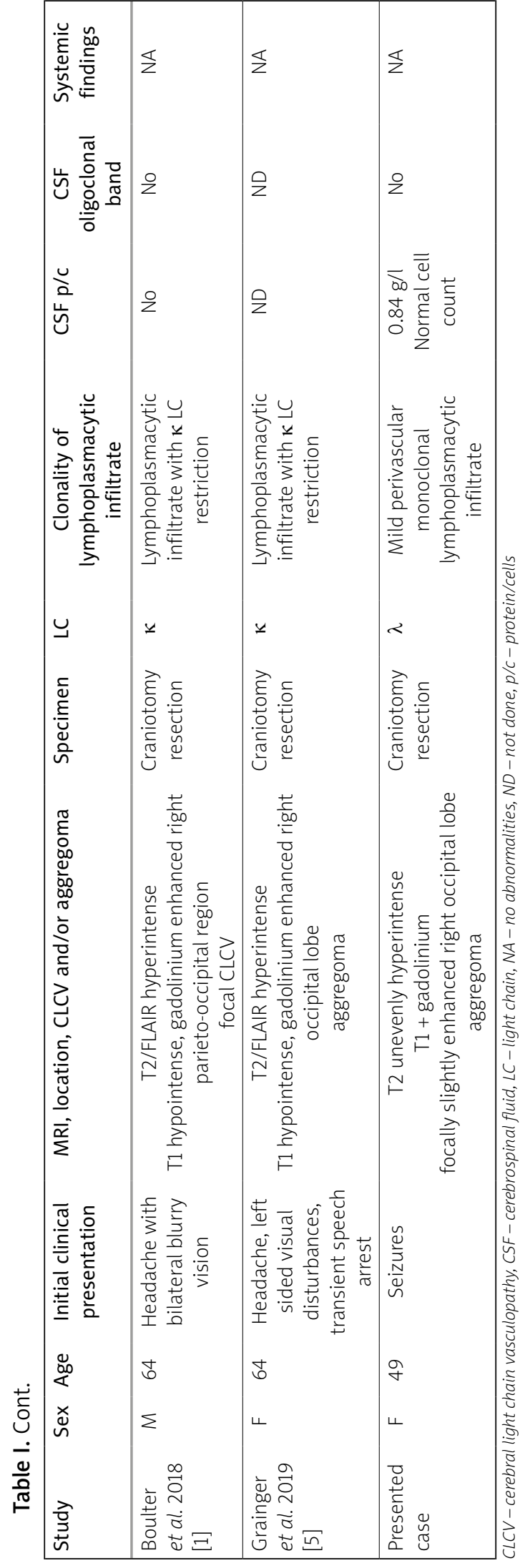

viously reported cases (Table I). The range of age at presentation was 19 to 72 years (mean age 52 years), which is only slightly younger than the age of appearance of primary central nervous system lymphomas (PCNSL) in immunocompetent patients [3], with a male predilection (male-to-female ratio $7: 4$ ). Another similarity between brain LCDD and PCNSL is the periventricular location in the majority of cases. In 7 cases, including the presented case, the lesion was located in the right occipital lobe close to the ventricle $[1,4,5,11,12,18]$. Even in the case with post-mortem brain examination, the largest lesion was in the same location [13]. In the other 3 cases, the locations were right cerebellar [17], right fronto-parietal [10], and right frontal [18]. The lesions were multifocal in 2 cases $[12,13]$. The leading symptom of disease in the presented case was epileptic seizure, as it was in the additional 2 with similar locations $[4,12]$. Otherwise, there were hemiparesis with or without hemihypesthesia $[10,11,15]$, headache with speech and visual disturbances [1,5], gait instability in the case with cerebellar location [17], and bulbar signs in the case with multifocal CLCV with severe medullary involvement [13]. The MRI presentation of all cases was very similar.

Specimens for microscopic observation of the lesions were obtained by stereotactic biopsy in 6 cases $[4,10-12,15,18]$, by craniotomy followed by excision of the lesion in 4 cases, including the presented one $[1,5,17]$, and whole brain examination was performed in 1 case [13]. In stereotactic biopsy specimens, the exact location of the aggregates could not always be determined, while in the excised specimens after craniotomy, the location of aggregates was well defined: in 3 cases, including the presented one, the aggregates were parenchymal, sparing the vessel walls [5,17], and giving tumour-like LCDD called aggregoma, while in 1 case the aggregates were only in the vessel walls [1], giving focal CLCV. Multifocal CLCV with no parenchymal aggregates was clearly observed only in the case of whole brain examination [13].

In the presented case, NGS clonality testing showed monoclonal B-cell lymphocytes. In all other cases, monoclonal lymphoplasmacytic infiltrate was observed with $\kappa$-LC restriction in 4 cases $[1,5,11,17]$, and $\lambda$-LC restriction in 2 cases $[10,15]$, in contrast to amyloidomas of CNS, in which almost exclusively $\lambda$-LC have so far been described [8]. LC restriction in the lymphoplasmacytic infiltrate was not mentioned in 
the other 3 cases $[4,12,13]$. In 3 cases, the infiltrate was defined as primary cerebral low-grade lymphoma $[4,12,15]$. Cerebrospinal fluid (CSF) examination was done in all cases except $2[5,10]$. CSF oligoclonal bands were found in only 2 cases $[12,13]$. A wholebody work up was performed in all cases except 1 [15], showing no sign of systemic immune dyscrasia.

To summarize, LCDD restricted to the brain is a very rare disease, radiologically mimicking a tumour or multiple sclerosis. We propose the name aggregoma only in cases with deposition of LC inside the brain tissue, with or without deposition in the vessel walls. The nature of monoclonal lymphoplasmacytic infiltrate, reactive or neoplastic, accompanying the deposits in all cases, remains questionable.

\section{Ethics approval}

The publication of the case report was approved by an institutional review board.

\section{Availability of data and material}

The data and the participation consent for the current case are not publicly available due to privacy concerns but are available from the corresponding author upon reasonable request.

\section{Acknowledgments}

The authors are grateful to Nina Hauptman, Ph.D. and Metod Perme for their technical support. Martin Cregeen provided language editing.

\section{Disclosure}

The authors report no conflict of interest.

\section{References}

1. Boulter TD, Sadaka A, Nakawah MO, Smith SV, Alouch N, Berry SE, Whyte AT, Fuller GN, Lee AG. Primary central nervous system lymphoma with light chain deposition disease (aggregoma). J Neuroophthalmol 2018; 38: 195-197.

2. Buxbaum JN, Chuba JV, Hellman GC, Solomon A, Gallo GR. Monoclonal immunoglobulin deposition disease: light chain and light and heavy chain deposition diseases and their relation to light chain amyloidosis. Clinical features, immunopathology, and molecular analysis. Ann Intern Med 1990; 112: 455-464.

3. Camilleri-Broet S, Martin A, Moreau A, Angonin R, Henin D, Gontier MF, Rousselet MC, Caulet-Maugendre S, Cuillière P, Lefrancq T, Mokhtari K, Morcos M, Broët P, Kujas M, Hauw JJ, Desablens B, Raphaël M. Primary central nervous system lymphomas in 72 immunocompetent patients: pathologic findings and clinical correlations. Groupe Ouest Est d'etude des Leucenies et Autres Maladies du Sang (GOELAMS). Am J Clin Pathol 1998; 110: 607-612.

4. Fischer L, Korfel A, Stoltenburg-Didinger G, Ransco C, Thiel E. A 19-yearold male with generalized seizures, unconsciousness and a deviation of gaze. Brain Pathol 2006; 16: 185-186, 187.

5. Grainger BT, Issa S, Anderson NE. Primary idiopathic CNS non-amyloidogenic light chain deposition disease complicated by treatment-resistant focal seizure disorder. J Clin Neurosci 2019; 59: 313-315.

6. Leonardis L, Pihler DN, Zupan M, Vizjak A, Belcijan NL, Jurcic V, Popovic $M$. Fatal recurrent dermatoneuro syndrome associated with systemic AL amyloidosis. Clin Neuropathol 2016; 35: 72-77.

7. Lighezan R, Baderca F, Alexa A, lacovliev M, Bonte D, Murarescu ED, Nebunu A. The value of the reprocessing method of paraffin-embedded biopsies for transmission electron microscopy. Rom J Morphol Embryol 2009; 50: 613-617.

8. Löhr M, Kessler AF, Monoranu CM, Grosche J, Linsenmann T, Ernestus RI, Härtig W. Primary brain amyloidoma, both a neoplastic and a neurodegenerative disease: a case report. BMC Neurol 2019; 19: 59

9. Matjasic A, Zupan A, Bostjancic E, Pizem J, Popovic M, Kolenc D. A novel PTPRZ1-ETV1 fusion in gliomas. Brain Pathol 2020; 30: 226-234.

10. Menke JR, Jentoft ME, Dogan A, Avent JM, Miller DV, Giannini C. Periventricular white matter immunoglobulin lambda light chain deposition disease diagnosed by proteomic analysis. Acta Neuropathol 2012; 124: 293-295.

11. Mercado JJ, Markert JM, Meador W, Chapman P, Perry A, Hackney JR. Primary CNS nonamyloidogenic light chain deposition disease: case report and brief review. Int J Surg Pathol 2017; 25: 755-760.

12. Pantazis G, Psaras T, Krope K, von Coelln R, Fend F, Bock T, Schittenhelm J, Melms A, Meyermann R, Bornemann A. Cerebral low-grade lymphoma and light chain deposition disease: exceedingly high IgG levels in the cerebrospinal fluid as a diagnostic clue. Clin Neuropathol 2010; 29: 378-383.

13. Popovic M, Tavcar R, Glavac D, Volavsek M, Pirtosek Z, Vizjak A. Light chain deposition disease restricted to the brain: The first case report. Hum Pathol 2007; 38: 179-184.

14. Rustad EH, Hultcrantz M, Yellapantula VD, Akhlaghi T, Ho C, Arcila ME, Roshal M, Patel A, Chen D, Devlin SM, Jacobsen A, Huang Y, Miller JE, Papaemmanuil E, Landgren O. Baseline identification of clonal V(D)J sequences for DNA-based minimal residual disease detection in multiple myeloma. PloS One 2019; 14: e0211600-e.

15. Skardelly M, Pantazis G, Bisdas S, Feigl GC, Schuhmann MU, Tatagiba MS, Tatagiba MS, Ritz R. Primary cerebral low-grade B-cell lymphoma, monoclonal immunoglobulin deposition disease, cerebral light chain deposition disease and "aggregoma": an update on classification and diagnosis. BMC Neurol 2013; 13: 107.

16. Toor AA, Ramdane BA, Joseph J, Thomas M, O'Hara C, Barlogie B, Walker P, Joseph L Cardiac nonamyloidotic immunoglobulin deposition disease. Mod Pathol 2006; 19: 233-237.

17. Vital A, Ellie E, Loiseau H. A 61-year-old man with instability of gait and right hand clumsiness. Brain Pathol 2010; 20: 273-274.

18. Yu JP, Wilson DM, Chang EF, Cotter J, Perry A, Mahindra A, Glastonbury CM. Isolated intracerebral light chain deposition disease: novel imaging and pathologic findings. Clin Imaging 2014; 38: 868-871.

19. Zidar N, Zver S, Jurcic V. Extraosseus plasmacytoma of the pharynx with localized light chain deposition. Case report. Pathol Oncol Res 2010; 16: 249-252. 